\title{
Support Theorem for Diffusion Processes on Hilbert Spaces
}

By

\author{
Shigeki AIDA*
}

\section{§1. Introduction}

In this paper we will prove a support theorem for infinite dimensional diffusion processes on Hilbert spaces. In finite dimensional cases we have a celebrated Stroock-Varadhan's theory ([1], [2]). We briefly review their theory. (See $\S 2$ for the below notation.)

Let $X(t)(0 \leqq t \leqq T)$ be the diffusion process governed by the following stochastic differential equation (abbreviation, SDE).

$$
\begin{aligned}
d X(t) & =\sigma(X(t)) \cdot d w(t)+b(X(t)) d t \\
X(t) & =x
\end{aligned}
$$

where $\sigma \in C_{b}^{2}\left(R^{n} \rightarrow R^{n} \otimes R^{m}\right), \quad b \in C_{b}^{1}\left(R^{n} \rightarrow R^{n}\right)$, and $w(t)$ is an $m$-dimensional Brownian motion. The notation $\cdot d w(t)$ denotes the Stratonovich stochastic differential. The problem is to determine the topological support of the diffusion measure $P_{x}$ of $X(t)$ which is a probability measure on $C_{x}\left([0, T], R^{n}\right)$ endowed with the uniform convergence topology. To prove the support theorem they first used the approximation theorem in the following. Let $\xi(\cdot, h)$ be the solution of the following ordinary differential equation (ODE),

$$
\begin{aligned}
& \dot{\xi}(t, h)=\sigma(\xi(t, h)) \dot{h}(t)+b(\xi(t, h)) \\
& \xi(0, x)=x
\end{aligned}
$$

where $h$ is a piecewise smooth function from $[0, T]$ to $R^{m}$ with $h(0)=0$ and let

$$
\begin{array}{r}
w^{k}(t)=\frac{2^{k}}{T}\left[\left\{t-\left((j-1) \frac{T}{2^{k}}\right)\right\} w\left(\frac{T}{2^{k}}(j)\right)+\left(\frac{T}{2^{k}} j-t\right) w\left(\frac{T}{2^{k}}(j-1)\right)\right] \\
\left(\frac{T}{2^{k}}(j-1) \leqq t \leqq \frac{T}{2^{k}} j\right) .
\end{array}
$$

Then $\xi\left(\cdot, w^{k}\right)$ converges to $X(t, w)$ uniformly as $k \rightarrow \infty$ a.s., which yields

Communicated by H. Araki, April 25, 1989.

* Mathematical Institute, Tohoku University, Sendai 980, Japan. 
$\operatorname{Supp}\left(P_{x}\right) \subset \overline{\mathscr{S}}_{x}$, where $\mathscr{S}_{x}=\left\{\xi(\cdot, h) \mid h \in C_{0}^{1}([0, T], E)\right\}$. The closure is taken with respect to the uniform convergence topology.

To prove the converse inclusion $\overline{\mathscr{S}}_{x} \subset \operatorname{Supp}\left(P_{x}\right)$ they considered the conditional probability $P_{\delta, h}^{\varepsilon}:=P\left(\|X(\cdot, w)-\xi(\cdot, h)\|_{T}>\varepsilon \mid\|w-h\|_{T}<\delta\right)$ where $\varepsilon$ $>0, \delta>0$, and $h \in C_{0}^{2}\left([0, T], R^{m}\right)$ and they showed $\lim _{\delta \rightarrow 0} P_{\delta h}^{\varepsilon}=0$, for every $\varepsilon>0$ and $h \in C_{0}^{2}\left([0, T], R^{m}\right)$. Since $P\left(\|w-h\|_{T}<\delta\right)>0$ for arbitrary $\delta>0$ and $h \in H_{0}^{1}\left([0, T], R^{m}\right)$, it implies that $\xi(\cdot, h) \in \operatorname{Supp}\left(P_{x}\right)$. Consequently, $\overline{\mathscr{S}}_{x}$ $=\operatorname{Supp}\left(P_{x}\right)$ holds.

In this formulation of the support theorem it should be noted that (1) is a Stratonovich's SDE. Stroock-Varadhan's procedure is not always valid, since the Stratonovich correction term (the difference between the Stratonovich integral and the Itô integral) diverges in general in infinite dimensional spaces. H. Doss [8] obtained the following sufficient condition under which the strong solution $X(\cdot, w)$ is continuous with respect to the uniform convergence topology of the Wiener space.

Theorem (H. Doss [8]). Let $\sigma \in C_{b}^{2}(E, L(B, E))$ and $b \in C_{b}^{1}(E, E)$. Assume further that

$$
D \sigma(x)\left(\sigma(x) h_{1}\right)\left(h_{2}\right)=D \sigma(x)\left(\sigma(x) h_{2}\right) h_{1} \quad \text { for every } \quad h_{1}, h_{2} \in H .
$$

Let $\xi$ be the solution of the following $O D E$.

$$
\begin{aligned}
& \dot{\xi}(t, x, h)=\sigma(\xi(t, h)) \dot{h}(t)-\frac{1}{2} \operatorname{trace} D \sigma(\xi(t, h))(\sigma(\xi(t, h))+b(\xi(t, h)) \\
& \xi(0, x, h)=x \quad h \in C_{0}^{1}([0, T], B) .
\end{aligned}
$$

Then $\xi: C_{0}^{1}([0, T], B) \rightarrow C([0, T], E)$ is continuous with respect to the uniform convergence topology of $C_{0}([0, T], B)$. Let us denote the continuous extension of this mapping by the same notation $\xi$ and denote by $X$ the strong solution of the following $S D E$.

$$
\begin{aligned}
d X(t) & =\sigma(X(t)) d W(t)+b(X(t)) d t \\
X(0) & =x .
\end{aligned}
$$

Then $X(t, x, W)=\xi(t, x, W)$.

Consequently Stroock-Varadhan's support theorem holds under (3) in infinite dimensional cases. However, we think that this result is too restrictive, since an important point of Stroock-Varadhan's support theorem is that the Wiener functional $X$ is not necessarily continuous, but is controlled by the ODE. In the present paper we will first assert that Stroock-Varadhan's arguments for the support theorem are valid even in infinite dimensional cases if one give a certain definition to the Stratonovich correction term quite well. Next, we will prove 
that if the diffusion generator is nondegenerate in a certain sense, then the support is the total space whether the Stratonovich correction term diverges or not.

The content of this paper is organized as follows. In $\S 2$ we will prepare some notions and several lemmas. In $\S 3$ we will prove our main theorem (Theorem 1), and in particular we will show the following:

Corollary 1 . Let $X(t)$ be the solution of the following $S D E$, and let $P_{x}$ denote the distribution of $X(t)$ on $C([0, T], E)$.

$$
\begin{aligned}
d X(t) & =\sigma(X(t)) d W(t)+b(X(t)) d t \quad(0 \leqq t \leqq T) \\
X(0) & =x
\end{aligned}
$$

Assume that

(1) $\sigma \in C_{b}^{4}(E, \mathscr{H}(H, E))$, and $b \in C_{b}^{1}(E, E)$,

(2) (a) $D \sigma \in C_{L i p}(E, \mathscr{H}(H \otimes E, E))$ or

(b) $\sigma \in C_{b}^{2}(E, L(B, E))$ holds.

Then $\frac{1}{2} \operatorname{trace} D \sigma(x)(\sigma(x))$ is well-defined, and

$$
\operatorname{Supp}\left(P_{x}\right)=\overline{\mathscr{S}}_{x}
$$

where $\mathscr{S}_{x}=\left\{\xi(\cdot, h) \mid h \in C_{0}^{1}([0, T], H)\right\}$, and $\xi$ is the solution of the following $O D E$ :

$$
\begin{aligned}
& \dot{\xi}(t, h)=\sigma(\xi(t, h)) \dot{h}(t)+b(\xi(t, h))-\frac{1}{2} \operatorname{trace} D \sigma(X(t)) \sigma(X(t)) \\
& \xi(0, h)=x,
\end{aligned}
$$

and the closure is taken with respect to the topology of $C([0, T], E)$.

In $\S 4$ we will prove a support theorem for nondegenerate diffusions. In $\S 5$ we will give several examples of $\mathrm{SDE}$ of which Stratonovich correction terms diverge, and discuss the support of them. In the last section we will prove the support theorem for stochastic flows, noting that stochastic flows are regarded as diffusion processes on a diffeomorphism group (Elworthy [7]).

\section{§2. Preliniminary}

Let $(\mu, H, B)$ be an abstract Wiener space, i.e. $B$ is a real separable Banach space and $H$ is a real separable Hilbert space continuous and densely embedded in $B$ and $\mu$ is a Gaussian measure such that for any $u \in B^{*}$ (dual space of $B$ ), $\int_{B} \exp \left(i_{B}\langle z, u\rangle_{B^{*}}\right) \mu(d z)=\exp \left(-\frac{1}{2}\|u\|_{H}^{2}\right)$, where $u \in B^{*} \subset H^{*} \simeq H$. See $\mathbb{H}$. $\mathbb{H}$. 
Kuo[4].

Let $W(t)$ be an $\mathscr{F}_{t}$-standard Wiener process on $B$ defined on a complete probability space $(\Omega, \mathscr{F}, P)$ with a right continuous filtration $\left\{\mathscr{F}_{t}\right\}$. For a Banach space $X,\|\|_{X}$ denotes its norm. We will often use \|\| instead of \|\|$_{X}$ if there is no confusion.

Throughout this paper, we will use the following notation. For any Banach spaces $X$ and $Y, L(X, Y)$ denotes the set of all bounded linear operators from $X$ to $Y$ and \|\|$_{L(X, Y)}$ denotes the uniform operator norm. For any separable Hilbert spaces $X$ and $Y, \mathscr{H}(X, Y)$ denotes the set of all HilbertSchmidt operators from $X$ to $Y$. \|\|$_{\mathscr{H}(X, Y)}$ denotes the Hilbert-Schmidt norm, and we use the notation $\mathscr{H}(X)$ if $X=Y$.

We will define function spaces. In the below definitions, $X$ and $Y$ stand for Banach spaces.

Let $C_{b}^{k}(X, Y)$ denote the Banach space consisting of all $k$-times continuously Fréchet differentiable maps from $X$ to $Y$ such that their derivatives are bounded up to the $k$-th order with the uniform convergence topology up to the $k$-th derivative. Let $C_{L i p}(X, Y)$ denote the set of all Lipschitz continuous functions from $X$ to $Y$. Let $C^{n}([0, T], X)$ denote the Banach space consisting of all continuously $n$-times Fréchet differentiable functions from $[0, T]$ to $X$ with the norm \|\|$_{T}$ given by $\|f\|_{T}=\sup _{0 \leqq t \leqq T} \sum_{k=0}^{n}\left\|f^{(k)}(t)\right\|_{X}$. In particular, we denote by $C_{x}^{n}([0, T], X)$ a subset of $C^{n}([0, T], X)$ with $f(0)=x$, where $x \in X$. Hereafter $E$ stands for a real separable Hilbert space.

We recall the definition of Itô's stochastic differential equations on Hilbert spaces. (See e.g. K. Itô [5], D. A. Dawson [6], H. H. Kuo [4]) Let $\sigma \in$ $C_{L i p}(E, \mathscr{H}(H, E))$ and $b \in C_{L i p}(E, E)$, and let us consider the following SDE.

$$
\begin{aligned}
d X(t) & =\sigma(X(t)) d W(t)+b(X(t)) d t \\
X(0) & =x \in E
\end{aligned}
$$

Here $\sigma(X(t)) d W(t)$ is the Itô stochastic differential. By usual method of Picard's succesive approximation, we can solve (2). To define a Storatonovich type SDE, we have to consider a correction term.

Definition 2.1. Let $\sigma \in C_{L i p}(E, \mathscr{H}(H, E))$ satisfying that $\sigma(x) h \in C_{b}^{1}(E, E)$ for every $h \in H$. For a complete orthonormal system (c. o. n. s.) $\left\{e_{i}\right\}_{i \geqq 1}$ in $H$, we say that $\sigma$ belongs to a class $S_{f}$ if the following conditions are satisfied.

(I) There exists $K>0$ such that

$$
\left\|V_{\sigma, f}^{n}(x)-V_{\sigma, f}^{n}(y)\right\|_{E} \leqq K\|x-y\|_{E} \text { for every } n \geqq 1 \text { and every } x, y \in E,
$$
where $V_{\sigma, f}^{n}(x):=\frac{1}{2} \sum_{i=1}^{n} D \sigma(x)\left(\sigma(x) e_{i}\right)\left(e_{i}\right)$.

(II) $\lim _{n \rightarrow \infty} V_{\sigma, f}^{n}(x)$ exists for every $x \in E$. 
We denote the limit by $V_{\sigma, f}(x)$. Then we see that $V_{\sigma, f} \in C_{L i p}(E, E)$. We will say $V_{\sigma, f}$ is the Stratonovich correction term and denote $V_{\sigma, f}$ by $\frac{1}{2} \operatorname{trace} D \sigma(x)(\sigma(x))$ if for every c.o.n.s. $f, \sigma \in S_{f}$ and $V_{\sigma, f}$ coincide with one another.

Remark (1) Note that $V_{\sigma, f}$ depends on the order of $f=\left\{e_{i}\right\}_{i=1}^{\infty}$. (2) We should notice that $V_{\sigma, f}$ is not well-defined even if $\sigma \in L(E, \mathscr{H}(H, E))$ in general (See Ex. 1 in $\S 5$ ).

Lemma 2.1. Let $\sigma \in C_{b}^{1}(E, \mathscr{H}(H, E))$. If $\sigma \in S_{f}$ for every c.o.n.s. $f$, then $V_{\sigma, f}$ coincide with one another.

Proof. It follows immediately from the following fact for the bounded linear operator: Let $A \in L(E)$ and assume that for every c.o.n.s. $\left\{e_{i}\right\}_{i}$, $\sum_{i=1}^{\infty}\left(A e_{i}, e_{i}\right)$ converges, then $A$ is uniquely decomposed to the following.

$$
A=A_{1}+A_{2},
$$

where $A_{1}$ is a bounded self adjoint operator of trace class and $A_{2}$ is a bounded skew symmetric operator. This is proved by taking $A_{1}=\left(A+A^{*}\right) / 2, A_{2}=$ $\left(A-A^{*}\right) / 2$, where $A^{*}$ is adjoint of $A$ and by using the fact in Reed-Simon [11] p. $218,26$.

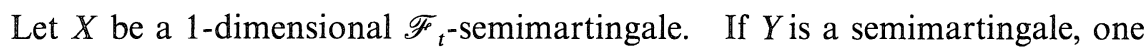
can define Stratonovich integral $\int Y(t) \cdot d X(t)$ as well as finite dimensional cases.

Definition 2.2. Let $X$ be a 1-dimensional $\mathscr{F}_{t}$-semimartingale, let $Y$ be as follows.

$$
Y(t)=Y(0)+\int_{0}^{t} \eta(s) d W(s)+\int_{0}^{t} \tau(s) d s
$$

where $\eta(t)$ is an $\mathscr{F}_{t}$-adapted $\mathscr{H}(H, E)$-valued process with

$$
E\left[\int_{0}^{T}\|\eta(t)\|^{2} d t\right]<\infty
$$

and $\tau(t)$ is an $\mathscr{F}_{t}$-adapted $E$-valued process with

$$
E\left[\int_{0}^{T}\|\tau(t)\| d t\right]<\infty
$$

It is easy to see that the following stochastic integral is well-defined.

$$
\int_{0}^{t} Y(t) \cdot d X(t):=\lim _{|\Delta| \rightarrow 0} \sum_{j=1}^{n} Y\left[\frac{t_{j+1}+t_{j}}{2}\right]\left(X\left(t_{j+1}\right)-X\left(t_{j}\right)\right) \text { a.s. }
$$


where $\Delta$ is a partition of $[0, t]$ i.e. $0<t_{1}<t_{2}<\cdots<t_{n}=t$, and

$$
|\Delta|=\max _{1 \leqq i \leqq n}\left(t_{i+1}-t_{i}\right)
$$

Then we have

Lemma 2.2. Let $Y$ be the same as in Definition 2.3, and set $w^{i}(t)$ $=\left(W(t), e_{i}\right)_{H}$. Then

$$
\int_{0}^{t} Y(s) \cdot d w^{i}(s)=\int_{0}^{t} Y(s) d w^{i}(s)+\frac{1}{2} \int_{0}^{t} \eta_{i}(s) d s
$$

where $\eta_{i}(s)=\eta(s) e_{i}$.

Lemma 2.3. Let $X$ be the solution of (2), and assume $\sigma \in S_{f}$. Then

$$
\begin{aligned}
\int_{0}^{t} g\left(X_{s}\right) \cdot d w^{i}(s)= & {\left[w^{i}(s) g\left(X_{s}\right)\right]_{0}^{t}-\sum_{j=1}^{\infty} \int_{0}^{t} D g\left(X_{s}\right)\left(V_{j}\left(X_{s}\right)\right) w^{i}(s) \cdot d w^{j}(s) } \\
& -\int_{0}^{t} w^{i}(s) D g\left(X_{s}\right)\left(b\left(X_{s}\right)-V_{\sigma, f}\left(X_{s}\right)\right) d s
\end{aligned}
$$

where $V_{i}(x):=\sigma(x) e_{i}, w^{i}(s)=\left(W(s), e_{i}\right)$, and $\left\{e_{i}\right\}=f$.

Proof. It is immediate from the Itô formula (see Elworthy[7]).

Remark. Let $\sigma(x) e_{i}=V_{i}(x)$. Then the $\operatorname{SDE}(2)$ is rewritten in the following form.

$$
\begin{aligned}
d X_{t} & =\sum_{i=1}^{\infty} V_{i}\left(X_{t}\right) d w_{i}(t)+b\left(X_{t}\right) d t \\
X_{0} & =x .
\end{aligned}
$$

We will use this form in the proof of Theorem 1.

In the rest of this section we summarize the basic facts which is used to prove Theorem 1 (see Stroock-Varadhan [1], [2] and Ikeda-Watanabe [10]).

Lemma 2.4. Let $w$ be a d-dimensional Brownian motion with $w(0)=0$.

Then $P\left(\|w\|_{T}<\delta\right) \sim C_{1} \exp \left(-C_{2} T / \delta^{2}\right)(\delta \rightarrow 0)$ where $C_{1}, C_{2}(>0)$ are constants. Here $f_{1}(t) \sim f_{2}(t)(t \rightarrow 0)$ means $\lim _{t \rightarrow 0} f_{1}(t) / f_{2}(t)=1$.

Lemma 2.5. Let $w=\left(w_{1}, w_{2}\right)(w(0)=0)$ be a two dimensional Brownian motion, and set $\eta^{i, j}(t)=\int_{0}^{t} w^{i}(s) \cdot d w^{j}(s)$. Then for every $\varepsilon>0 \quad \delta>0 \quad P\left(\left\|\eta^{i, j}\right\|_{T}\right.$ $\left.\geqq \varepsilon \mid\|w\|_{T} \leqq \delta\right) \leqq C_{3} \exp \left(-\left(\frac{\varepsilon}{\delta}\right)^{2} \frac{C_{4}}{T}\right)$ where $C_{3}, C_{4}(>0)$ are absolute constants and $P(\cdot \mid \cdot)$ stands for the conditional probability. 
We need the following estimate to prove Theorem 1.

Lemma 2.6. Let us consider the following continuous $L^{2}$-martingale $M(t)$ on Hilbert space E:

$$
M(t)=\int_{0}^{t} \sigma(s, \omega) d W(s)
$$

where $\sigma(s, \omega)$ is a $\mathscr{H}(H, E)$ valued $\mathscr{F}_{t}{ }^{\text {-optional process such that }}$

$$
\|\sigma(s, \omega)\|_{\mathscr{H}(H, E)} \leqq K \text { a.s. } \quad \text { for }(s, \omega) .
$$

Then

$$
P\left(\|M\|_{T} \geqq R\right) \leqq A \exp \left\{-\frac{B}{T}\left(\frac{R}{K}\right)^{2}\right\},
$$

where $A, B$ are absolute constants.

Proof. It is sufficient to prove the lemma when $R=1$. By the Itô formula we have

$$
\|M(t)\|^{2}=2 \int_{0}^{t}\left(\sigma(s)^{*} M(s), d W(s)\right)_{H}+\int_{0}^{t}\|\sigma(s)\|_{\mathscr{H}(H, E)}^{2} d s
$$

where $\sigma(s)^{*}$ is adjoint of $\sigma(s)$.

Note that $P\left(n \leqq\|M\|_{T} \leqq n+1\right)$

$$
\begin{aligned}
& \leqq P\left(n^{2}-K^{2} T \leqq\left\|2 \int_{0}^{t}\left(\sigma(s)^{*} M(s), d W(s)\right)_{H}\right\|_{T} \leqq(n+1)^{2}+K^{2} T,\right. \\
& \left.\|M\|_{T} \leqq n+1\right) \\
& \leqq P\left(\left\|2 \int_{0}^{t}\left(\sigma(s)^{*} M(s \wedge \tau), d W(s)\right)_{H}\right\|_{T} \geqq n^{2}-K^{2} T\right) \\
& \leqq 2 \exp \left[-\frac{\left(n^{2}-K^{2} T\right)^{2}}{8(n+1)^{2} K^{2} T}\right] \\
& \leqq 2 \exp \left[-\frac{1}{8 K^{2} T} \cdot \frac{n^{4}}{(n+1)^{2}}\right] \cdot \exp \left[\frac{n^{2}}{4(n+1)^{2}}\right] \\
& \leqq 2 \exp \left(-\frac{n}{32 K^{2} T}\right) \cdot \exp \left(\frac{1}{4}\right) \text {. }
\end{aligned}
$$

Here $\tau=\inf \{t \geqq 0 \mid\|M(t)\|>n+1\}$.

$$
P\left(\|M\|_{T} \geqq 1\right)=\sum_{n=1}^{\infty} P\left(n \leqq\|M\|_{T}<n+1\right)
$$




$$
\leqq 2 \exp \left(\frac{1}{4}\right) \frac{\exp \left(-\frac{1}{32 K^{2} T}\right)}{1-\exp \left(-\frac{1}{32 K^{2} T}\right)}
$$

Hence $P\left(\|M\|_{T} \geqq 1\right) \leqq 8 \exp \left(-\frac{1}{32 K^{2} T}\right)$, which implies that Lemma 2.6 holds with $A=8, B=1 / 32$.

\section{§3. Proof of the Main Theorem}

Theorem 1. Let $X$ be the solution of the following SDE, and let $P_{x}$ be the distribution of $X(t)$ on $C([0, T], E)$.

$$
\begin{aligned}
& d X(t)=\sigma(X(t)) d W(t)+b(X(t)) d t \\
& X(0)=x
\end{aligned}
$$

Assume that

(1) $\sigma \in C_{L i p}(E, \mathscr{H}(H, E))$, and $b \in C_{b}^{1}(E, E)$.

(2) there exists a c.o.n.s $f=\left\{e_{i}\right\}$ of $H$ such that the following $(a)$ or $(b)$ are satisfied:

(a) $\sigma(x) e_{i} \in C_{b}^{4}(E, E)$

(b) there exists $A_{i} \in \mathscr{H}(E, E)$ and $\varphi_{i} \in C_{b}^{3}(E, E)$ such that $\sigma(x) e_{i}=\varphi_{i}\left(A_{i} x\right)$.

(3) for the same c.o.n.s. f, $\sigma \in S_{f}$ holds.

Then,

$$
\operatorname{Supp}\left(P_{x}\right)=\overline{\mathscr{S}}_{x}
$$

where $\mathscr{S}_{x}=\left\{\xi(\cdot, h) \mid h \in C_{0}^{1}([0, T], E)\right\}$ and $\xi$ is the solution of the following ODE:

$$
\begin{aligned}
& \dot{\xi}(t, h)=\sigma(\xi(t, h)) \dot{h}(t)+b(\xi(t, h))-V_{\sigma, f}(\xi(t, h)) \\
& \xi(0, h)=x
\end{aligned}
$$

The closure is taken with respect to the topology of $C([0, T], E)$.

Proof. The proof of $\operatorname{Supp}\left(P_{x}\right) \subset \mathscr{S}_{x}$ is immediate from the approximation theorem. We omit the proof since it is essentially the same as Elworthy [7] p. 104. We will prove the opposite direction by adopting Stroock-Varadhan's method. Without loss of generality we may assume that $\sigma \in C_{b}(E, \mathscr{H}(H, E))$, and $V_{\sigma, f} \in C_{b}(E, E)$. We first claim the following.

Lemma 3.1. For an $h=\left(h^{i}(t)\right)_{i=1}^{\infty} \in C_{0}^{\infty}\left([0, T], \ell^{2}\right)$, we set

$$
h_{n}(t):=\left(h^{1}(t), \cdots, h^{n}(t)\right),
$$




$$
\begin{aligned}
& w_{n}(t):=\left(w^{1}(t), \cdots, w^{n}(t)\right), \\
& P_{\delta, h_{n}}(\cdot)=P\left(\cdot \mid\left\|h_{n}-w_{n}\right\|_{T}<\delta\right), \text { and } \\
& P_{x, h_{n}}^{\delta}(\cdot)=P_{\delta, h_{n}}(X(\cdot, x, \omega) \in \cdot) .
\end{aligned}
$$

Note that $P_{x, h_{n}}^{\delta}$ is a probability measure on $C([0, T], E)$. Then

(1) $\lim _{\delta \rightarrow 0} P_{x, h_{n}}^{\delta}=P_{x, h_{n}}^{0}$ holds weakly for each $n \geqq 1$, where $P_{x, h_{n}}^{0}$ is a probalility measure on $C([0, T], E)$ induced by the solution $X_{n, h}(t)$ of the following $S D E$.

$$
\begin{aligned}
d X(t) & =\sum_{i=1}^{n} V_{i}(X(t)) \dot{h}^{i}(t) d t+\sum_{j=n+1}^{\infty} V_{j}(X(t)) d w^{j}(t)+\left\{b(X(t))-V_{\sigma, f}^{n}(X(t))\right\} d t \\
X(0) & =x .
\end{aligned}
$$

(2) Let $X_{\infty, h}$ be the solution of the following $O D E$.

$$
\begin{aligned}
& \dot{X}(t)=\sum_{i=1}^{\infty} V_{i}(X(t)) \dot{h}^{i}(t)+\left\{b((t))-V_{\sigma, f}(X(t))\right\} \\
& X(0)=x .
\end{aligned}
$$

Then $\lim _{n \rightarrow \infty} E\left[\left\|X_{n, h}-X_{\infty, h}\right\|_{T}^{2}\right]=0$.

Using Lemma 3.1 we can complete the proof of Theorem 1. Because, by Lemma $3.1 \lim _{\delta \rightarrow 0} P_{x, h_{n}}^{\delta}=P_{x, h_{n}}^{0}$ and $\lim _{n \rightarrow \infty} P_{x, h_{n}}^{0}=\delta_{X_{\infty, h}}$ hold weakly, where $\delta_{X_{\infty, h}}$ is the Dirac measure at $X_{\infty, h}$ and $\operatorname{Supp}\left(P_{x, h_{n}}^{\delta}\right) \subset \operatorname{Supp}\left(P_{x}\right)$, we have $\operatorname{Supp}\left(\delta_{X_{\infty, h}}\right)$ $\subset \operatorname{Supp}\left(P_{x}\right)$ which implies $X_{\infty, h} \in \operatorname{Supp}\left(P_{x}\right)$. To prove Lemma 3.1 (1), we need the following lemma.

Lemma 3.2. For every $R>0,0<\delta<1, \alpha>1$

$$
P_{\delta, h_{n}}\left(\left\|\int_{0}^{t} V_{i}(X(s)) \cdot d w^{i}(s)-\int_{0}^{t} V_{i}(X(s)) \dot{h}^{i}(s) d s\right\|_{T} \geqq R\right) \leqq\left(\frac{\delta}{R}\right)^{\alpha} C_{\alpha}
$$

where $C_{\alpha}$ is a constant which is independent of $R$ and $\delta$.

Proof. By the Cameron-Martin formula we may assume $h=0$. First we prove the lemma under the condition (a) of Theorem 1. By applying Lemma 2.3, we have

$$
\begin{gathered}
\int_{0}^{t} V_{i}(X(s)) \cdot d w^{i}(s)=\left[V_{i}(X(s)) w^{i}(s)\right]_{0}^{t}-\sum_{j=1}^{\infty} \int_{0}^{t} D V_{i}(X(s))\left\{V_{j}(X(s))\right\} w^{i}(s) \\
\cdot d w^{j}(s)-\int_{0}^{t} D V_{i}(X(s))\left\{b(X(s))-V_{\sigma, f}(X(s))\right\} w^{i}(s) d s .
\end{gathered}
$$


Further applying Lemma 2.3 repeatedly, we get

$$
\begin{aligned}
& \int_{0}^{t} V_{i}(X(s)) \cdot d w^{i}(s)=\left[P_{i}(s) w_{i}(s)+\sum_{j=1}^{n} Q_{i, j}(s) \eta^{i, j}(s)+\sum_{j, k=1}^{n} R_{i, j, k}(s) w^{i}(s) \eta^{i, j}(s)\right]_{0}^{t} \\
& +\int_{0}^{t}\left(w^{i}(s) \alpha_{i}(s)+\sum_{j=1}^{n} \beta_{i, j}(s) \eta^{i, j}(s)+\sum_{j, k=1}^{n} \gamma_{i, j, k}(s) w^{i}(s) \eta^{i, j}(s)\right) d s \\
& +\sum_{k=n+1}^{\infty} \int_{0}^{t}\left(w^{i}(s) \Phi_{i, k}(s)+\sum_{j=1}^{n} \eta^{i, j}(s) \Psi_{i, j, k}(s)\right) d w^{k}(s)+\int_{0}^{t} \sum_{m=1}^{n} F_{m}(s) d w^{m}(s) \\
& \quad=I_{1}+I_{2}+I_{3}+I_{4} .
\end{aligned}
$$

Here $\eta^{i, j}(t)=\int_{0}^{t} w^{i}(s) \cdot d w^{j}(s)$, and $P_{i}, Q_{i, j}, R_{i, j, k}, \alpha_{i}, \beta_{i, j}$ and $\gamma_{i, j, k}$ are $E$-valued bounded continuous $\mathscr{F}_{t}$-stochastic processes involving $V_{j}(j \geqq 1)$ and up to their fourth derivatives. $\Phi_{i, j}$ and $\Psi_{i, j, k}$ are $E$-valued continuous $\mathscr{F}_{t}$-stochastic processes such that $\sum_{k=n+1}^{\infty}\left(\left\|\Phi_{i, k}(s)\right\|_{E}^{2}+\sum_{j=1}^{\infty}\left\|\Psi_{i, j, k}(s)\right\|_{E}^{2}\right) \leqq C<\infty$, where $C>0$ is a constant independent of $\omega$.

$$
F_{m}(s)=\sum_{j, k=1}^{n} a_{m, i, j, k}(s) w^{i}(s) \eta^{j, k}(s)+\sum_{j=1}^{n} b_{m . i, j}(s) w^{i}(s) w^{j}(s)
$$

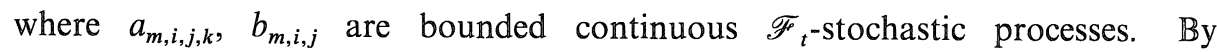
Lemma 2.4 and Lemma 2.5 and the martingale inequality we have easily that for every $R>0$, every $\delta(0<\delta<1)$ and every $\alpha>1$,

$$
P_{\delta, n}\left(\left\|I_{1}+I_{2}+I_{3}\right\|_{T} \geqq R\right) \leqq C_{\alpha}\left(\frac{\delta}{R}\right)^{\alpha} .
$$

Therefore we need only to prove the following. For every $R>0$ and $\delta(0<\delta$ <1) $P_{\delta, n}\left(\left\|I_{4}\right\|_{T}>R\right) \leqq C \exp \left(-\frac{C^{\prime}}{\delta} R\right)$, where $C$ and $C^{\prime}(>0)$ are constants independent of $R$ and $\delta$.

$$
\begin{aligned}
P_{\delta, n}\left(\left\|I_{4}\right\|_{T} \geqq R\right)= & \frac{P\left(\left\|I_{4}\right\|_{T} \geqq R,\left\|w_{n}\right\|_{T} \leqq \delta\right)}{P\left(\left\|w_{n}\right\|_{T} \leqq \delta\right)} \\
\leqq & \frac{P\left(\left\|I_{4}\right\|_{T} \geqq R,\left\|w_{n}\right\|_{T} \leqq \delta, \sum_{j, k=1}^{n}\left\|\eta_{j, k}\right\|_{T}<\sqrt{R \delta}\right)}{P\left(\left\|w_{n}\right\|_{T} \leqq \delta\right)} \\
& +\frac{P\left(\sum_{j, k=1}^{n}\left\|\eta_{i, j}\right\|_{T} \geqq \sqrt{R \delta}\right)}{P\left(\left\|w_{n}\right\|_{T} \leqq \delta\right)}
\end{aligned}
$$




$$
\leqq \frac{A \exp \left[-\frac{B}{T}\left(\frac{R}{\sqrt{R \delta} \delta C}\right)^{2}\right]}{C_{1} \exp \left(-\frac{C_{2}}{\delta^{2}} T\right)}+C_{4} \exp \left(-\frac{R}{\delta} \frac{C_{5}}{T}\right)
$$

where $A, B, C$ and $C_{i}$ are constants independent of $R$ and $\delta$. At last inequality we used Lemma 2.4, Lemma 2.5 and Lemma 2.6. This completes the proof of Lemma 3.2 under the assumption (a) of Theorem 1.

Next we prove the lemma under the condition (b) of Theorem 1 . We need the following lemma.

Lemma 3.3. Let $(\mu, H, B)$ be an abstract Wiener space. Let us denote $C_{b}^{r}(H, X)_{B}:=\left\{f \in C_{b}^{r}(H, X) \mid\right.$ there exists a $\hat{f} \in C_{b}^{r}(B, X)$ such that $\left.\left.\hat{f}\right|_{H}=f\right\}(r \geqq 1)$. For every $f \in C_{b}^{r}(H, X)_{B}$ there exists a sequence $\left\{f_{m}\right\}_{m=1}^{\infty} \in C_{b}^{\infty}(H, X)$ such that $\lim _{m \rightarrow \infty} f_{m}=f$ with respect to the topology of $C_{b}^{r-1}(H, X)$.

This can be proven by using molifier as well as finite dimensional cases. See H. H. Kuo [4] p. 146 Theorem 6.1.

Proof of Lemma 3.2 under the condition (b). By combining the similar argument to Stroock-Varadhan [1] p. 351, and the above proof, we need only to prove the following. For every $i$ and $j$, there exists $\left\{U_{i, j}^{n}\right\}_{n=1}^{\infty} \in C_{b}^{\infty}(E, E)$ such that

$$
\lim _{n \rightarrow \infty} U_{i, j}^{n}=D V_{i}(x)\left(V_{j}(x)\right) \text { in the topology of } C_{b}^{1}(E, E)
$$

To prove this we notice that $V_{i}$ satisfies the assumption of Lemma 3.3. Set $E_{i}$ $=\overline{\left(\left(\operatorname{Ker} A_{i}\right)^{\perp},(,)_{i}\right)} \oplus\left(\operatorname{Ker} A_{i},(,)_{E}\right)$ where $\oplus$ stands for the direct sum of Hilbert spaces and $(\cdot, \cdot)_{i}=\left(A_{i} \cdot A_{i} \cdot\right)$ and the closure is taken with respect to this norm. Then a natural Hilbert-Schmidt embedding $l: E \rightarrow E_{i}$ exists. By Minlos-Sazonov's theorem, $\left(E, E_{i}\right)$ with Gaussian measure $\mu$ is an abstract Wiener space. Clearly $V_{i}(x)=\varphi_{i}\left(A_{i} x\right) \in C_{b}^{3}\left(E_{i}, E\right)$. This implies that $V_{i}$ satisfies the assumption of Lemma 3.3 with respect to an abstract Wiener space $\left(\mu, E, E_{i}\right)$ with $r=2$, which completes the proof.

Now we are in position to prove Lemma 3.1.

Proof of Lemma 3.1. By Lemma 3.2

$$
\lim _{\delta \rightarrow 0} E^{P_{x}^{\delta}, h_{n}}\left[\left\|\sum_{i=1}^{n} \int_{0}^{t} V_{i}(X(s)) \cdot d w^{i}(s)-\sum_{i=1}^{n} \int_{0}^{t} V_{i}(X(s)) \dot{h}^{i}(s) d s\right\|_{T}^{2}\right]=0 .
$$

Note that 


$$
\begin{aligned}
X(t)-X_{n, h}(t)= & \sum_{i=1}^{n} \int_{0}^{t}\left(V_{i}(X(s)) \cdot d w^{i}(s)-V_{i}(X(s)) \dot{h}^{i}(s)\right) d s \\
& +\sum_{i=1}^{n} \int_{0}^{t}\left\{V_{i}(X(s))-V_{i}\left(X_{n, h}(s)\right)\right\} \dot{h}^{i}(s) d s \\
& +\sum_{j=n+1}^{\infty} \int_{0}^{t}\left\{V_{j}(X(s))-V_{j}\left(X_{n, h}(s)\right)\right\} d w^{j}(s) \\
& +\int_{0}^{t}\left\{U_{\sigma, f}^{n}(X(s))-U_{\sigma, f}^{n}\left(X_{n, h}(s)\right)\right\} d s,
\end{aligned}
$$

where $U_{\sigma, f}^{n}(x):=b(x)-V_{\sigma, f}^{n}(x)$. By a standard argument we have

$$
\begin{aligned}
& E^{P_{x}^{\delta}, h_{n}}\left[\left\|X-X_{n, h}\right\|_{t}\right] \leqq E^{P_{x}^{\delta}, h_{n}}\left[\left\|\int_{0}^{t} V_{i}\left(X_{s}\right) \cdot d w_{s}^{i}-\int_{0}^{t} V_{i}\left(X_{s}\right) \dot{h}_{s}^{i} d s\right\|_{T}\right] \\
& +K \cdot \int_{0}^{t} E^{P_{x, h_{n}}^{\delta}}\left[\left\|X-X_{n, h}\right\|_{s}\right] d s, \quad(K>0) \text {. }
\end{aligned}
$$

Using Gronwall inequality we get Lemma 3.1 (1).

(2) It also follows from the Gronwall inequality, so we will omit the proof.

Remark. It would be plausible that Theorem 1 holds under the condition that $V_{i} \in C_{b}^{2}(E, E)$ in place of (2), since under this condition the approximation theorem still holds, so $\operatorname{Supp}\left(P_{x}\right) \subseteq M$ follows. If one can prove that a family of the probability measure $\left\{P_{x, h_{n}}^{\delta}\right\}_{\delta>0}$ is tight, one can prove the support theorem under this condition by the above method.

Next we will prove Corollary 1.

Proof. (a) Let $f=\left\{e_{i}\right\}$ be an arbitrary c.o.n.s. of $H$.

$$
\begin{aligned}
\left\|\sum_{i=n}^{m} D \sigma(x)\left(\sigma(x) e_{i}\right)\left(e_{i}\right)\right\| & \leqq \sum_{i=n}^{m}\left\|D \sigma(x)\left(\sigma(x) e_{i}\right)\left(e_{i}\right)\right\| \\
& \leqq \sum_{i=n}^{m}\left\|D \sigma(x)(\cdot) e_{i}\right\|_{\mathscr{H}(E)}\left\|\sigma(x) e_{i}\right\|_{E} \\
& \leqq\left\|D \sigma(x)(\cdot)\left(P_{m}-P_{n}\right)\right\|_{\mathscr{H}(H \otimes E, E)}^{2}\left\|\sigma(x)\left(P_{n}-P_{m}\right)\right\|_{\mathscr{H}(H, E)}^{2},
\end{aligned}
$$

where $P_{n}$ denotes the projection on the linear span of $\left\{e_{i}\right\}_{i=1}^{n}$. Thus we have $\lim _{n \rightarrow \infty} \sum_{i=1}^{n} D \sigma(x)\left(\sigma(x) e_{i}\right)\left(e_{i}\right)$ exists. This implies the well-definedness of trace $D \sigma(x)(\sigma(x))$ by Lemma 2.1. By the similar argument (also by using Lipschitz continuity of $D \sigma)$ we have easily that there exists $K(>0)$ for arbitrary $n$ 


$$
\left\|\sum_{i=1}^{n} D \sigma(x)\left(\sigma(x) e_{i}\right)\left(e_{i}\right)-\sum_{i=1}^{n} D \sigma(y)\left(\sigma(y) e_{i}\right)\left(e_{i}\right)\right\| \leqq K\|x-y\|
$$

which implies $\sigma \in S_{f}$.

(b) We will freely use the standard facts in measure theory on Banach spaces (H. H. Kuo [4]). For any c.o.n.s. $f=\left\{e_{i}\right\}$ of $H$,

$$
\begin{aligned}
\lim _{n \rightarrow \infty} \sum_{i=1}^{n} D \sigma(x)\left(\sigma(x) e_{i}\right)\left(e_{i}\right) & =\lim _{n \rightarrow \infty} \int_{B} D \sigma(x)\left(\sigma(x) P_{n} z\right)\left(P_{n} z\right) \mu(d z) \\
& =\int_{B} D \sigma(x)(\sigma(x) z)(z) \mu(d z)
\end{aligned}
$$

where $P_{n}$ is the same as of (a), and at the last equality we used the following results :

(I) $D \sigma(x)(\sigma(x) \cdot)(\cdot)$ is bounded bilinear form on $B \times B$

(II) $\|z\|_{B},\left\|P_{n} z\right\|_{B} \in L^{p}(B, d \mu)(p \geqq 1)$ and $\lim _{n \rightarrow \infty} \int_{B}\left\|P_{n} z-z\right\|_{B}^{p} \mu(d z)=0$

(c.f. H.H.Kuo [4], p. 82, Theorem 4.5 for (II)).

By similar arguments we have

$$
\begin{aligned}
& \left\|\sum_{i=1}^{n} D \sigma(x)\left(\sigma(x) e_{i}\right)\left(e_{i}\right)-\sum_{i=1}^{n} D \sigma(y)\left(\sigma(y) e_{i}\right)\left(e_{i}\right)\right\| \\
& \quad \leqq \int_{B}\left\|D \sigma(x)\left(\sigma(x) P_{n} z\right)\left(P_{n} z\right)-D \sigma(y)\left(\sigma(y) P_{n} z\right)\left(P_{n} z\right)\right\|_{E} \mu(d z) \\
& \quad \leqq \int_{B} K\|x-y\|_{E}\left\|P_{n} z\right\|_{B}^{2} \mu(d z)
\end{aligned}
$$

which completes the proof.

\section{§4. Support Theorem for Nondegenerate Diffusions}

In this section, we will prove a support theorem for non-degenerate diffusions. We will first give the following definition.

Definition 4.1. Let $\sigma \in C(E, \mathscr{H}(H, E))$. We say that $\sigma$ is nondegenerate if the following condition is satisfied.

(C) There exists a dense subspace $F$ of $E$ such that

$$
F \subset \operatorname{Im} \sigma(x) \text { for all } x \in E \text {. }
$$

When $\sigma$ satisfies the assumption (C) with $F$, we will say that $\sigma$ is nondegenerate with respect to $F$.

Theorem 2. Let $X(t)$ be the solution of the following $S D E$. 


$$
\begin{aligned}
d X(t) & =\sigma(X(t)) d W(t)+b(X(t)) d t \quad(0 \leqq t \leqq T) \\
X(0) & =x .
\end{aligned}
$$

Assume that $\sigma$ satisfies the conditions (1) and (2) in Theorem 1 and $\sigma$ is nondegenerate, then

$$
\operatorname{Supp}\left(P_{x}\right)=C_{x}([0, T], E) .
$$

Proof. Without loss of generality, we may assume $\sigma \in C_{b}(E, \mathscr{H}(H, E))$. Let $\sigma$ be nondegenerate with respect to $F$. Let $Q_{n}$ be the finite dimensional projection onto the subspace $E_{n}$, where $\left\{E_{n}\right\}$ is increasing subspaces of $F$ such that $\bar{U}_{n} E_{n}=E$ and let $\left\{P_{n}\right\}_{n=1}$ be the increasing sequence of finite dimensional projections of $H$ such that $P_{n} \rightarrow I_{H}$ strongly. First we will show the following claim.

(Claim) Let $X_{n, m}(t)$ be the solution of the following SDE.

$$
\begin{aligned}
d X_{n, m}(t)= & \sigma_{n}\left(X_{n, m}(t)\right) \cdot d W_{n}(t)+\bar{\sigma}_{n}\left(X_{n, m}(t)\right) d \bar{W}_{n}(t) \\
& -\frac{1}{2}\left(I-Q_{m}\right)\left\{t r D \sigma_{n}\left(X_{n, m}(t)\right)\left(\sigma_{n}\left(X_{n, m}(t)\right)-2 b\left(X_{n, m}(t)\right)\right\} d t\right. \\
X_{n, m}(0)= & X .
\end{aligned}
$$

Here $\sigma_{n}(x)=\sigma(x) P_{n}, \bar{\sigma}_{n}(x)=\sigma(x)\left(I-P_{n}\right)$,

$$
W_{n}(t)=P_{n} W(t), \bar{W}_{n}(t)=W(t)-W_{n}(t) .
$$

Then two diffusion measures $P_{x}^{n, m}$ induced by $X_{n, m}$ and $P_{x}$ are mutually absolutely continuous. We will prove the claim. One can take a increasing sequence $\left\{K_{j}\right\}_{j}$ of compact subsets of $E$ such that

(*) let $\tau_{j}^{n, m}$ denote the first exit time from $K_{j}$ of $X$ or $X_{n, m}$, then $\lim _{j \rightarrow \infty} \tau_{j}^{n, m}=\infty$ $P$-a.s.

Comparing (1) with (2), we know that (2) has an extra drift term $\frac{1}{2} Q_{m}\left\{\operatorname{tr} D \sigma_{n}(x)\left(\sigma_{n}(x)\right)-2 b(x)\right\}$. By using the nondegeneracy of $\sigma$, we see that there exists neighborhood $U_{j}$ of $K_{j}(j \in N)$ and $C_{b}^{1}$ vector field $V_{j}$ on $E$ such that $\sigma(x) V_{j}(x)=\frac{1}{2} Q_{m}\left\{\operatorname{tr} D \sigma_{n}(x)\left(\sigma_{n}(x)\right)-b(x)\right\}$ on $U_{j}$. Therefore by the Girsanov formula and (*), we get the claim. By the claim we have Supp of $X_{n, m}=\operatorname{Supp}$ of $X$. Letting $m \rightarrow \infty$, we get from the Gronwall inequality argument

$$
\lim _{m \rightarrow \infty} E\left[\left\|X_{n, m}-X_{n}\right\|_{T}^{2}\right]=0,
$$

where $X_{n}$ is the solution of the following SDE. 


$$
\begin{aligned}
d X_{n}(t) & =\sigma_{n}\left(X_{n}(t)\right) \cdot d W_{n}(t)+\bar{\sigma}_{n}\left(X_{n}(t)\right) d \bar{W}_{n}(t) \\
X_{n}(0) & =x .
\end{aligned}
$$

Clearly Support of $X_{n} \subset$ Support of $X$.

By a similar argument of Lemma 3.2, we can prove the following.

$$
\lim _{\delta \rightarrow 0} E_{x, h_{n}}^{P_{x}^{\delta}}\left[\left\|\int_{0}^{t} \sigma_{n}\left(X_{n}(s)\right) \cdot d W_{n}(s)-\int_{0}^{t} \sigma_{n}\left(X_{n}(s)\right) \dot{h}^{n}(s) d s\right\|_{T}\right]=0 .
$$

By the same argument as the proof of Lemma 3.1 (2) we have $\xi(t, h) \in \operatorname{Supp}\left(P_{x}\right)$, where $\xi(t, h)$ is the solution of the following ODE.

$$
\begin{aligned}
& \dot{\xi}(t, h)=\sigma(\xi(t, h)) \dot{h}(t) \quad h \in C_{0}^{\infty}([0, T], H) \\
& \xi(0, h)=x .
\end{aligned}
$$

This completes the proof.

\section{§5. Examples}

In this section, we will discuss below three examples. For Example 1, Theorem 1 and Theorem 2 are not applicable. For Example 2, Theorem 1 is not applicable but Theorem 2 is applicable. For Example 3, Theorem 1 and Theorem 2 are not applicable but we can characterize its support. Hereafter we set $E=H=\ell^{2}$. Therefore every Hilbert-Schmidt operator is canonically identified with a matrix of which matrix elements are square summable. We here restrict ourselves to the following SDE.

$$
d X(t)=a_{k}(X(t)) d W(t) \quad(k=1,2,3)
$$

where $a_{k}(x)$ is chosen in the below examples respectively.

Example 1. Let $\varphi$ be a bijective map from $Z^{2^{+}}=\left\{(i, j) \in Z^{2} \mid i, j \geqq 1\right\}$ onto $N$. Associated with $\varphi$, we define a bounded linear operator $a_{1}: H \rightarrow \mathscr{H}(H)$ as follows;

$$
\left(a_{1}(x)(y)\right)_{i}=\sum_{j=1}^{\infty} x_{\varphi(i, j)} y_{j}
$$

We can calculate its Stratonovich correction term easily.

If $e_{i}=\left[\begin{array}{c}0 \\ \vdots \\ 1 \\ \vdots \\ 0\end{array}\right] i$ then 
$i$-th component of $V_{a_{1}}^{n}(x)$ is $\frac{1}{2} \sum_{j=1}^{n} x_{\varphi(\varphi(i, j), j)}$.

Clearly, this quantity diverges for every $x$ in a dense subset of $\ell^{2}$ converges in another dense subset. Also for any other c.o.n.s., the Stratonovich correction term diverges in a dense subset and converges in another dense subset. Therefore our theorems are not applicable. It is interesting problem to determine the support of this diffusion process. But we do not know how to characterize the support at present.

Example 2. Let

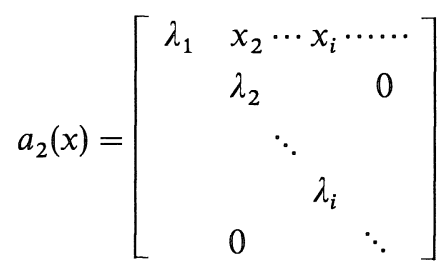

where $\left\{\lambda_{i}\right\}_{i} \in \ell^{2}$. In this case, for $\left\{e_{i}\right\}_{i}$ (see Example 1)

$$
V_{a_{2}}^{n}(x)=\frac{1}{2}\left[\begin{array}{c}
\sum_{i=2}^{n} \lambda_{i} \\
0 \\
0 \\
\vdots
\end{array}\right] .
$$

Therefore the Stratonovich correction term diverges in general. But if $\lambda_{i} \neq 0$ for every $i$, then by Theorem 2 the support is total space. This example is due to S. Kusuoka.

Example 3. Let us consider a variation of the above Example 2 as follows:

$$
a_{3}(x)=\left[\begin{array}{cccc}
x_{2} & \cdots & x_{i+1} & \cdots \cdots \\
\lambda_{1} & & & \\
& \ddots & & 0 \\
& & \lambda_{i} & \\
& 0 & \ddots &
\end{array}\right] \text { where }\left\{\lambda_{i}\right\}_{i} \in \ell^{2} .
$$

If $\sum_{i=1}^{\infty} \lambda_{i}$ converges, we have the following characterization of $P_{x}$.

$$
\begin{aligned}
& \operatorname{Supp}\left(P_{x}\right)=\overline{\mathscr{S}}_{x}, \text { where } \mathscr{S}_{x}=\left\{\xi(\cdot, h) \mid h \in C_{0}^{\infty}\left([0, T], \ell^{2}\right)\right\} \\
& \xi(t, h)=\sum_{i=1}^{\infty} \lambda_{i} \frac{h^{i}(t)^{2}-t}{2} e_{1}+\sum_{i=1}^{\infty} \lambda_{i} h^{i}(t) e_{i+1}+x
\end{aligned}
$$


In general cases though our theorem is not applicable, we can see its support in the following way.

Lemma 5.1. Let $E$ be a normed space, and let $\left\{X_{i}\right\}_{i=1}^{\infty}$ be E-valued independent random variables. If $S:=\sum_{i=1}^{\infty} X_{i}$ converges in $L^{1}$ sence, we have $\operatorname{Supp}(S)=\bigcap_{m=1}^{\infty} \overline{\bigcup_{n=m}^{\infty} M_{n}}$, where $M_{n}=\sum_{i=1}^{n} \operatorname{Supp}\left(X_{i}\right)$, and $\operatorname{Supp}(S)$ and $\operatorname{Supp}\left(X_{i}\right)$ denote the topological support of the image measures of $S$ and $X_{i}$ respectively.

Proof. $\operatorname{Supp}(S) \subseteq \bigcap_{m=1}^{\infty} \overline{\bigcup_{n=m}^{\infty} M_{n}}$ is clear. We will prove the opposite direction. Let $x \in \bigcap_{m=1}^{\infty} \overline{\bigcup_{n=m}^{\infty} M_{n}}$. For every $\varepsilon>0$, there exists $n_{0} \in N$ such that $E\left[\sum_{i=n_{0}}^{\infty}\left\|X_{i}\right\|_{E}\right] \leqq \varepsilon$. By the assumption of $x$, there exists $x_{i} \in \operatorname{Supp}\left(X_{i}\right)$ $\left(i=1,2, \cdots n, n \geqq n_{0}\right)$ such that $\left\|\sum_{i=1}^{n} x_{i}-x\right\| \leqq \varepsilon$. Since $\left\{X_{i}\right\}$ are independent, we have

$$
\begin{aligned}
& E\left[\|S-x\|_{E} \mid \sum_{i=1}^{n}\left\|X_{i}-x_{i}\right\|_{E}<\varepsilon\right] \\
& \quad \leqq E\left[\sum_{i=1}^{n}\left\|X_{i}-x_{i}\right\|_{E}+\left\|\sum_{i=1}^{n} x_{i}-x\right\|+\sum_{i=n+1}^{\infty}\left\|X_{i}\right\|_{E} \mid \sum_{i=1}^{n}\left\|X_{i}-x_{i}\right\|_{E}<\varepsilon\right] \\
& \quad \leqq 3 \varepsilon .
\end{aligned}
$$

This implies $x \in \operatorname{Supp}(S)$.

We will return to Example 3. The solution of (1) has the following explicit solution. This is used in characterization of $(*)$.

$$
\begin{aligned}
& X_{1}(t)=\frac{1}{2} \sum_{i=1}^{\infty} \lambda_{i}\left(w_{i}(t)^{2}-t\right)+x_{1} \\
& X_{i}(t)=\lambda_{i-1} w_{i-1}(t)+x_{i}(i \geqq 2),
\end{aligned}
$$

where $X_{i}(t)=\left(X(t), e_{i}\right)$,

i.e.

$$
X(t)=\sum_{i=1}^{\infty} Y_{i}(t)+x
$$

where $Y_{i}(t)=\frac{1}{2} \lambda_{i}\left(w_{i}(t)^{2}-t\right) e_{1}+\lambda_{i} w_{i}(t) e_{i+1}$. Therefore by Lemma 4.1, we can characterize the support of $X(t)$ as follows:

$$
\operatorname{Supp}(X(\cdot))=\bigcap_{m=1}^{\infty} \overline{\bigcup_{n=m}^{\infty} M_{n}},
$$


where $M_{n}=\left\{\xi^{n}(\cdot, h) \mid h \in C_{0}^{\infty}\left([0, T], R^{n}\right)\right\}$ and

$$
\xi^{n}(\cdot, h)=\sum_{i=1}^{n}\left\{\frac{1}{2} \lambda_{i}\left(h^{i}(t)^{2}-t\right) e_{1}+\lambda_{i} h^{i}(t) e_{i+1}\right\}+x .
$$

\section{\$6. Support Theorem for Stochastic Flows}

We will apply our theorem to stochastic flows. We refer to Elworthy [7], Le Jan-Watanabe [9] about stochastic flows. In particular we use the notation of Elworthy [7].

Theorem 3. Let $M$ be a compact manifold of dimension d. Let $\left\{V_{i}\right\}_{i=0}^{\infty}$ be vector fields on $M$ satisfying the following conditions

$$
V_{i} \in H^{s+2}(T M)(i \geqq 1) \text { and } \sum_{i=1}^{\infty}\left\|V_{i}\right\|_{H^{s+2}(T M)}^{2}<\infty,
$$

$$
V_{0} \in H^{s+1}(T M)
$$

where $s \geqq 1+\frac{1}{2} d$. Let $\varphi(t)$ be the stochastic flow on $\mathscr{D}^{s}(M)$ governed by the following SDE.

$$
\begin{aligned}
d X(t, x, \omega) & =\sum_{i=1}^{\infty} V_{i}(X(t, x, \omega)) \cdot d w^{i}(t)+V_{0}(X(t, x, \omega)) d t \\
X(0, x, \omega) & =x .
\end{aligned}
$$

Let $P$ be the probability of $\varphi(t)$ on $C\left([0, T], \mathscr{D}^{s}(M)\right)$. Then Supp $(\mathbb{P})=\overline{\mathscr{S}}_{x}$, where $\mathscr{S}_{x}=\left\{\xi(\cdot, h) \mid h \in C^{1}\left([0, T], \ell^{2}\right)\right\}$ and $\xi(\cdot, h)$ is the solution of the following ODE:

$$
\begin{aligned}
& \dot{\xi}(t, h, x)=\sum_{i=1}^{\infty} V_{i}\left(\xi(t, h, x) \dot{h}(t)+V_{0}(\xi(t, h, x))\right. \\
& \xi(0, x, h)=x .
\end{aligned}
$$

The closure is taken with respect to the topology of $C\left([0, T], \mathscr{D}^{s}(M)\right)$.

Proof. We will show how to apply our theorem. First we will prove the theorem under the condition that $V_{i} \in H^{s+4}(T M)(i \geqq 1)$. By Elworthy [7], $\varphi(t)$ is the solution of the following $\mathrm{SDE}$ on $\mathscr{D}^{s}(M)$.

$(*) \quad d \bar{X}(t)=\left(\left(R_{\bar{X}(t)^{*}}\right) \cdot K\right) \cdot d W(t)+\bar{V}_{0}(\bar{X}(t)) d t$

$$
\bar{X}(0)=i d \text {, }
$$

where $R$ is the right translation, and $(R)_{*}$ is derivation of $R$, and $\bar{V}_{0}(\phi)$ : $=\left(\mathbb{R}_{\phi}\right)_{*} V_{0}$, and $K$ is a Hilbert-Schmidt operator on $\mathscr{H}\left(H^{s+2}(T M)\right)$ such that $K e_{i}$ $=V_{i} \quad(i \geqq 1)$. By the same reason as (b) of Corollary 1 we can write down a 
Stratonovich's $\operatorname{SDE}(*)$ according to stochastic flow $\varphi(t)$. Applying Theorem 1 to $(*)$, we get Theorem 3 under the $H^{s+4}$ condition. Next we relax the condition $H^{s+4}$ to $H^{s+2}$. There exists a sequence of $C^{\infty}$ vector fields $U_{i}^{n}(i \geqq 1)$ such that $\lim _{n \rightarrow \infty} U_{i}^{n}=V_{i}$ in $H^{s+2}(T M)$, which implies $\bar{V}_{i}$ can be approximated by the elements of $C^{\infty}\left(T \mathscr{D}^{s}(M)\right)$ in $C^{2}\left(T \mathscr{D}^{s}(M)\right)$, where $\bar{V}_{i}:=(R)_{*} V_{i}$. Therefore by the same argument as Theorem 1 (b), we get Theorem 3.

\section{Ackmowledgement}

The author is grateful to Professor T. Shiga and Professor M. Motoo for their hearty encouragements and valuable comments. The author is also grateful to Professor S. Kusuoka for fruitful discussions.

\section{Referemces}

[1] Stroock, D. W. and Varadhan, S.R.S., On the support of diffusion processes with applications to the strong maximal principle, Proc. of Sixth Berkeley Symp. Math. Statist. Prob., 㯰., 333-359, Univ. California Press, Berkeley, 1972.

[2] On Degenerate Elliptic-Parabolic Operators of Second Order and Their Associated Diffusions, Comm. Pure. Appl. Math., 25 (1972), 651-713.

[3] Stroock, D. W., An introduction to the Theory of Large Deviations, Universitext, SpringerVerlag, 1984.

[4] Kuo, H. H., Gaussian measures in Banach spaces, Lect. notes in Math. 463, Springer, 1975.

[5] Itô, K., Foundations of Stochastic Differential Equations in Infinite Dimensional Spaces, Regional Conference Series in Applied Mathematics 47, 1984.

[6] Dawson, D. A., Stochastic evolution equation and related measure process, J. Multivariate Anal., 5 (1975), 1-55.

[7] Elworthy, K. D., Stochastic Differential Equations on Manifolds, Cambridge Univ. Press, 1982.

[ 8 ] Doss, H., Liens entre' equations differentielles stochastiques et ordinaires, Ann. Inst. Henri Poincaré 13 (1977), 99-125.

[9] Le Jan, Y. -Watanabe, S., Stochastic flows of diffeomorphism, Proc. of Taniguchi Intern. Symp. on stochastic analysis, Katata \& Kyoto, ed. by K. Itô, Kinokuniya (1982), 307-332.

[10] Ikeda, N. -Watanabe, S., Stochastic Differential Equations and Diffusion Processes, North Holland, Amsterdam/Kodansha, Tokyo, 1981.

[11] Reed-Simon, Functional analysis, Academic press, 1972. 
